\title{
Robust optimization of machining parameters based on edge theorem
}

\author{
Deng Congying ${ }^{1,2}$, Feng $\mathrm{Yi}^{1}$, Ma Ying ${ }^{1}, \mathrm{Wei} \mathrm{Bo}^{1}$ and Miao Jianguo ${ }^{3, \text { a }}$ \\ ${ }^{1}$ School of Advanced Manufacturing Engineering, Chongqing University of Posts and Telecommunications, Chongqing 400065, PR \\ China \\ ${ }^{2}$ College of Mechanical Engineering, Chongqing University, Chongqing 400030 \\ ${ }^{3}$ Chongqing Midea Universal Refrigeration Equipment Co., Ltd, 15 Rose Road, Nan'an District, Chongqing 401336, PR China
}

\begin{abstract}
Traditional mathematic models for predicting milling stability assume that dynamic parameters of machine tools remain constant. However, these parameters such as natural frequencies and cutting force coefficients vary under operational state, reducing accuracies of the chatter prediction and related machining parameters optimization. In this study, the edge theorem and the zero exclusion condition are used to extend the traditional stability model for considering the effects of the uncertain parameters. Thus, robust combinations of the spindle speed and axial cutting depth are predicted. They are the inputs of the optimization model to obtain the maximum material remove rate MMR based on the particle swarm optimization method. The proposed machining parameters optimization method was applied on a real vertical machining center, and its effectiveness was validated by the chatter tests.
\end{abstract}

\section{Introduction}

Chatter is a self-induced vibration phenomenon caused by the variation of chip thickness under operational conditions. Its occurrence leads to poor surface finish, low productivity, increased tool wear, machine tool failure and so on [1-3]. Normally, the developed stability lobe diagram SLD technique is applied to predefining the appropriate chatter-free combinations of the machining parameters [4]. Thus, how to select the optimal combination of the machining parameters for reducing tool vibration and increasing the MMR plays an important role in the practical manufacturing engineering.

The machining parameters optimization has been researched in detail for decades [5-7]. In the optimization model established by $\mathrm{Li}$ et al [8], they studied the constraints including force, machine tool and tool motion. Cao et al [9] took the MMR as the objective, but its mechanical model was not suitable for high speed milling. Budak and Tekeli [10] proposed a method to determine the optimal combination of axial and radial depths of cut based on the two stability lobe diagrams. Fratila et al. [11] used Taguchi method to optimize the combination of cutting speed, feed rate and cutting depths to obtain the best workpiece processing quality. However, in a real machining application, the deviations between the measurements and predictions are still observed. This problem may be caused by the assumption that system dynamics (machine, tool, and workpiece) and machining conditions are constant and time invariant. However, these dynamic parameters such as natural frequencies and cutting force coefficients vary depending upon different aspects including spindle speeds, tool wear, and machining positions, reducing the accuracy of chatter prediction. Chatter stability prediction with uncertainties has already been addressed in recent researches [12]. But its application on machining parameters optimization is addressed little.

This paper presents a new method to obtain the optimal machining parameters based on the robust prediction of milling stability. Henceforth, the remainder of this paper is organized as follows: a description of the edge theorem and the zero exclusion condition for the robust chatter prediction is presented in Section 2. And the mathematic optimization model and the application of the particle swarm algorithm are provided in Section 3. This is followed by the case study and results discussion to verify the proposed method through experimental procedure in Section 4. Finally, conclusions from the current research are summarized in Section 5.

\section{Milling model with uncertainties}

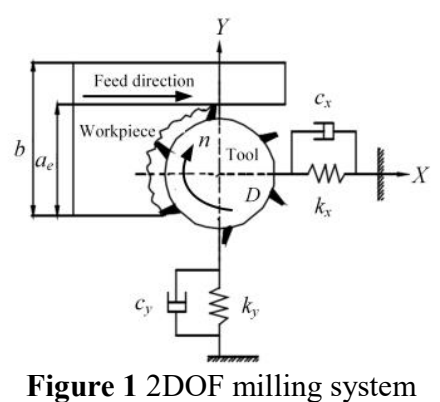

*Corresponding author: jianguo.miao@ midea.com 
Normally, a 2DOF milling system shown in Fig.1 is usually used in analytical chatter stability prediction. The general equations of motion for the 2DOF milling system can be expressed as follow:

$$
\left\{\begin{array}{l}
\Delta F_{x}(t) \\
\Delta F_{y}(t)
\end{array}\right\}=\frac{a_{p} K_{t c} N}{4 \pi}\left[\begin{array}{lll}
a_{x x}{ }^{1} & a_{x y}{ }^{1} \\
a_{x y} & { }^{1} & a_{y y}
\end{array}\right]\left\{\begin{array}{l}
\Delta x \\
\Delta y
\end{array}\right\}=\frac{a_{p} K_{t c} N}{4 \pi}\left[A_{1}\right]\left\{\begin{array}{l}
\Delta x \\
\Delta y
\end{array}\right\}
$$

Where $a_{p}$ is the axial cutting depth, $K_{t c}$ is the tangential cutting force coefficient, and $\left[\boldsymbol{A}_{1}\right]$ is average directional factors. Eq. (1) is transformed into the frequency domain:

$$
\{\boldsymbol{F}\} e^{i \omega t}=\frac{a_{p} K_{t c} N}{2 \pi}[\boldsymbol{A}]\left(1-e^{-i \omega_{c} \tau}\right)\left[\boldsymbol{G}\left(i \omega_{c}\right)\right]\{\boldsymbol{F}\} e^{i \omega_{c} t}
$$

$\boldsymbol{G}\left(i \omega_{c}\right)$ is the transfer function matrix in $x$ and $y$ direction, which is usually represented by the tool tip FRFs. The characteristic equation of Eq. (3) is:

$$
\operatorname{det}\left|\boldsymbol{I}+\Lambda\left[\boldsymbol{G}_{1}\left(i \omega_{c}\right)\right]\right|=0
$$

Where

$$
\begin{aligned}
& \Lambda=-\frac{a_{p} K_{t c} N}{4 \pi}\left(1-e^{-i \omega_{c} \tau}\right)=\Lambda_{I}+\Lambda_{R} \\
& {\left[\boldsymbol{G}_{1}\left(i \omega_{c}\right)\right]=\left[\begin{array}{ll}
a_{x x} & a_{x y} \\
a_{x y} & a_{y y}
\end{array}\right]\left[\begin{array}{ll}
G_{x x}\left(i \omega_{c}\right) & G_{x y}\left(i \omega_{c}\right) \\
G_{y x}\left(i \omega_{c}\right) & G_{y y}\left(i \omega_{c}\right)
\end{array}\right]}
\end{aligned}
$$

Then, the axial critical cutting depth $a_{\text {plim }}$ can be determined as

$$
a_{p \lim }=-\frac{2 \pi \Lambda_{R}\left[1+\left(\Lambda_{I} / \Lambda_{R}\right)^{2}\right]}{K_{t c} N}
$$

Eqs. (1) to (5) shows that the milling stability is mainly dependent on the tool tip FRFs and force coefficients. In the theoretical model, these parameters are assumed to be constant. However, they will change in real machining. Thus, effects caused by the uncertain parameters should be considered in machining parameters optimization.

\subsection{Stability prediction with uncertain parameter}

Based on the traditional stability model stated in section 2.1 , the edge theorem is adopted to consider variations of the tool tip FRFs and force coefficients. These parameters vary within their minimums and maximums respectively.

According to the edge theorem [13], if a polynomial $P$ with uncertainties, the combinations of extreme values will form the family of polynomials. Evaluating these extreme polynomials in a given frequency range, vertices will be obtained in the complex plane. If the edges between each pair of vertices are stable, then the system is robustly stable [13]. For high speed milling, Eq. (3) is transformed into another form to meet the polynomial form by ignoring the effects of the cross FRFs:

$$
a_{0} \Lambda^{2}+a_{1} \Lambda+1=0 \leftarrow \begin{aligned}
& a_{0}=G_{x x} G_{y y}\left(a_{x x} a_{y y}-a_{x y} a_{y x}\right) \\
& a_{1}=a_{x x} G_{x x}+a_{y y} G_{y y}
\end{aligned}
$$

To establish an accurate stability prediction model for optimizing machining parameters, modal parameters of tool tip FRFs in $x$ and $y$ directions and the radial and tangential force coefficients are taken as the uncertainties. Thus, the milling system polynomial $\boldsymbol{P}$ is:

$$
\boldsymbol{P}=\left\{p_{1}(s, \boldsymbol{v}), p_{2}(s, \boldsymbol{v}), \cdots, p_{i}(s, \boldsymbol{v})\right\}
$$

Where $i$ is the number of equations. If the number of uncertainties is $k, i$ equals $2^{k}$. And $v$ contains the variables, which can be express as follow:

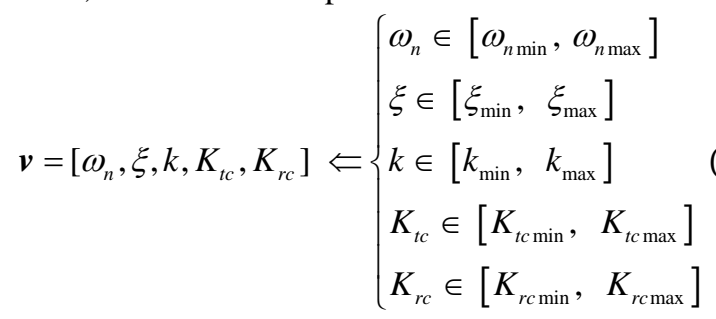

The zero exclusion principle states a graphical technique to evaluate the stability of the edges efficiently. It states that if the zero of the complex plane is not located within the polygon generated by forming edges between each vertex, the system is stable. A four-sided polygon shown in Fig. 2 is taken as an instance. Therefore, the chatter stability prediction is transformed into a graphical problem. An algorithm is developed to obtain the conservative stability lobe and its procedure is summarized in Fig. 3. For a given cutting depth and spindle speed, a range of frequencies is determined, and the extreme polygon at each frequency is calculated and graphically checked. If stable for all frequencies, the cutting depth is increased, and the aforementioned procedure is repeated until an unstable point is detected. The related cutting depth is recorded as the limit value. Then the algorithm moves onto the next spindle speed to detect another unstable cutting depth. Thus, for each combination of spindle speed, cutting depth and frequency, the system stability is detected until the focused spindle speed range is swept.
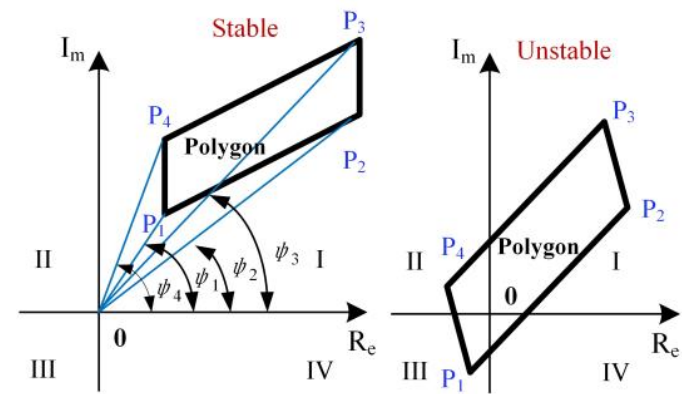

Figure 2 A four-side polygon 


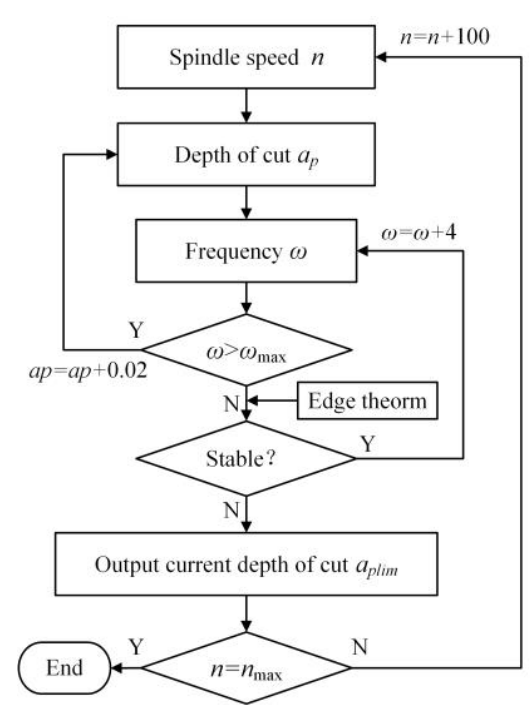

Figure 3 The flow chart of the chatter prediction

\section{Milling stability optimization}

The objective functions of the milling stability optimization are usually the maximum productivity, minimum processing cost, maximum economic profit and so on. In this paper, the purpose of the optimization is to obtain a higher productivity. Thus, the objective is the maximum material remove rate MMR, which can be expressed as Eq. (9):

$$
\operatorname{MMR}=n \cdot a_{p} \cdot a_{e} \cdot f_{z} \cdot N
$$

Despite the tooth number of the cutter $N$, the MMR is mainly determined by the main machining parameters including spindle speed $n$, feed per tooth $f_{v}$, axial cutting depth $a_{p}$ and radial cutting width $a_{e}$. In the optimization, $f_{v}$ and $a_{e}$ are regarded as constants. Then, the variables are the spindle speed $n$ and axial cutting depth $a_{p}$. Since the machining process must under a stable condition, these variables should meet the machining stability constraint that $a_{p}$ is smaller than the limiting axial cutting depth $a_{\text {plim. }}$. In addition, the constraints also include the spindle speed, torque and power of the machine tool, which can be expressed as:

$$
\begin{gathered}
T_{c}=F_{t} \cdot \frac{d}{2 \times 1000} \leq T_{\max } \\
P=F_{t} \cdot \frac{v_{c}}{60 \times 1000} \leq P_{\max } \cdot \eta \\
C_{1}(x)=v_{c}-\frac{n_{\max } p d}{60 \times 1000} \leq 0 \\
C_{2}(x)=\frac{n_{\min } p d}{60 \times 1000}-v_{c} \leq 0
\end{gathered}
$$

Where $n_{\min }$ and $n_{\max }$ are the minimal and maximum spindle speed, $p$ is the maximum power of the machine tool, and $\eta$ is the effective coefficient.

Thus, the machining stability optimization can be summarized as the following mathematic model:

$$
\begin{cases}\max & \mathrm{MMR}=n \cdot a_{p} \cdot a_{e} \cdot f_{z} \cdot N \\ \text { s.t. } & a_{p}<a_{p \lim } \\ & \text { cons }\end{cases}
$$

Considering the uncertainties in real machining process, the limiting axial cutting depths are determined by the algorithm aforementioned in section 2.2. Thus the spindle speed is discretized to obtain the corresponding limiting axial cutting depths $a_{p l i m}$. For one spindle speed, the axial cutting depth varies between 0 and $a_{\text {plim }}$. Then the combination of the spindle speed and axial cutting depths are used to conduct the optimization.

Since the particle swarm optimization algorithm PSO has faster convergence speed and higher accuracy [14], it is introduced to obtain the optimal resolution of the machining parameters. In D-dimensional space, $N$ particles are assumed to form a community. Characteristics of each particle are represented by the position $\boldsymbol{X}_{i}=\left(x_{i 1}, x_{i 2}, \ldots, x_{i D}\right)$, velocity $\boldsymbol{V}_{i}=\left(v_{i 1}, v_{i 2}, \ldots, v_{i D}\right)$ and fitness value $f_{i}$. Positions of these particles represent potential solutions to the optimization. The fitness value of each solution is evaluated based on the objective function to determine the optimal position. After one iteration, the particle best and the global best labeled as $P_{\text {best }}$ and $G_{\text {best }}$ are updated respectively. The two optimums are used to determine the velocities to modify the positions of particles in next iteration as follow:

$$
\left\{\begin{array}{l}
v_{i d}^{k+1}=w v_{i d}^{k}+c_{1} r_{1}\left(P_{\text {best }_{i d}}^{k}-x_{i d}^{k}\right)+c_{2} r_{2}\left(G_{\text {best }_{i d}}^{k}-x_{i d}^{k}\right) \\
x_{i d}^{k+1}=x_{i d}^{k}+v_{i d}^{k+1}
\end{array}\right.
$$

Where $w$ is the inertia weight, $k$ is the iterative number, $c$ is the velocity weighting factors equaling 0 or positive constant, and $r$ is the random numbers between 0 and 1 .

\section{Case study}

\section{1 machining stability prediction}

The above described technique to achieve optimal machining parameters considering the uncertainties were applied to a real three-axis vertical machining center. In the case studies, the tool and the workpiece were first selected. Then the impact testing were conducted on the tool tip to obtain the FRFs in $x$ and $y$ directions when the machine tool was under stationary state. Since it is difficult to obtain the tool tip FRFs under operational state, the change rates of the modal parameters belonging to the spindle are used to simulate the change rates of the modal parameters belonging to the tool tip. Then the obtained modal parameters and their variations are shown in Table 1.

Table 1. Modal parameters and their variations.

\begin{tabular}{|c|c|c|c|c|c|c|}
\hline & \multicolumn{3}{|c|}{ Frequency (Hz) } & \multicolumn{3}{c|}{ Damping ratios (\%) } \\
\hline mode & 1 & 2 & 3 & 1 & 2 & 3 \\
\hline$x$ & 482 & 786 & 972 & 2.71 & 3.56 & 2.64 \\
\hline$y$ & 657 & 794 & 1205 & 3.49 & 2.43 & 1.72 \\
\hline $\begin{array}{c}\text { Variat } \\
\text { ion } \\
(\%)\end{array}$ & -3.2 & -4.6 & -2.9 & -6.4 & -8.1 & -5.7 \\
\cline { 2 - 7 } & -4.1 & -2.1 & -7.3 & -8.6 & -6.4 \\
\hline
\end{tabular}


The cutting force coefficients were identified through the cutting test on end milling ASTM 1045. The tooth number is four, and the radial immersion and feed values were set as constants with the values of $6 \mathrm{~mm}$ and $0.02 \mathrm{~mm} /$ tooth. The nominal tangential and radial values are the cutting force coefficients 1963MPa and $742 \mathrm{MPa}$ respectively, and a $\pm 5 \%$ variation was determined considering the tool wear, thermal effects and so on. The spindle speed is discretized by $100 \mathrm{rpm}$. Thus, with the founded system parameters and their variations, the robust chatter stability lobe was plotted shown in Fig. 4.

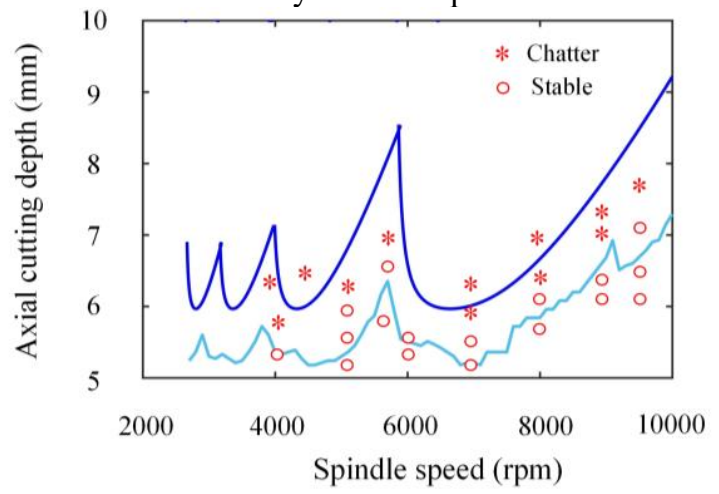

Figure 4 The robust predicted SLD

\section{2 machining parameters optimization}

For the PSO algorithm, the number of the particle swarm was initialized as 50, and each particle had two dimensions representing the spindle speed and axial cutting depth. The spindle speed varies between 0 and $15000 \mathrm{rpm}$ and its limiting axial cutting depth were calculated through the interpolation operation on the obtained discrete data in section 4.1. The initial value $w_{\max }$ and $w_{\min }$ were 0.9 and $0.4, c_{1}$ and $c_{2}$ were both set as 2 , and the number of iterations was 400 . The particles' initial positions and velocities were set randomly and further updated based on Eq (12). The updates depended on the fitness values calculated from the MMR through Eq. (9). Thus, after each iteration, the higher fitness values were used to update the particle best and global best, and then the iteration moved on to another. When the number of iterations met the upper limit, the final global best was the optimal machining parameters.

The obtained optimal spindle speed is $9408 \mathrm{rpm}$ and the axial cutting depth is $6.37 \mathrm{~mm}$. Chatter test were performed to verify the stability prediction considering the uncertainties. During the test, the acquired sound information was utilized to determine whether the chatter had occurred. Results of the chatter test are outlined in Fig. 3, in which the boundary of the robust SLD is below the boundary of the conventional SLD. The machining parameters below the boundary of the robust SLD (including the optimal spindle speed and axial cutting depth) all corresponds to the stable condition. However, the stable and unstable points both exist in the region between the robust and conventional SLD, which means that the chatter predicting considering the uncertainties have higher accuracy in real application.

\section{Conclusion}

The traditional stability model assumes that the dynamic parameters of the system are constants. However, in actual milling process, variations in machine tool dynamic parameters and cutting force coefficients will affect the prediction accuracy of machining stability. As a consequence, the optimization of machining parameters based on the traditional stability model will also show deviations from the real machining. Thus, the edge theorem and the zero principle are introduced to extend the traditional stability model to consider the uncertain parameters. The nominal values of the modal parameters and cutting force coefficients and their deviations are determined. These parameters are used to conduct the robust stability prediction. Then the robust combinations of spindle speed and axial cutting depth are applied into the mathematic optimization model to obtain the optimal combination with the maximum MMR based on the PSO algorithm. Chatter test were performed to verify the robust stability prediction, which also included the machining with the optimal combination. Results shows that the predicted robust SLD has a higher accuracy than the conventional SLD, and the proposed machining parameters optimization method can lay a foundation to the process planning.

\section{Acknowledgements}

This research is supported by the National Natural Science Foundation of China under Grant no. 51705058, the Science and Technology Research Program of Chongqing Municipal Education Commission under Grant no. KJ1704087 \& KJ1704072, and the Chongqing Research Program of Basic Research and Frontier Technology under Grant no. cstc2017jcyjAX0005.

\section{References}

1. J. Tlusty, M. Polacek, The stability of machine tools against self-excited vibrations in machining, Proc. ASME Int. Res. Prod. Eng. (1963) 465-474.

2. Y. Altintas, E. Budak, Analytical prediction of stability lobes in milling, Ann. CIRP 44 (1995)

3. Y. Altintas, G. Stepan, D. Merdol, Z. Dombovari, Chatter stability of milling in frequency and discrete time domain, Cirp J. Manuf. Sci. Technol., 1 (2008)

4. O. Özşahin, E. Budak, H.N. Özgüven. In-process tool point FRF identification under operational Conditions using inverse stability solution, Int. J. Mach. Tools Manuf., 89 (2014) 64-73.

5. X.M. Zhang, H. Ding. Note on a novel method for machining parameters optimization in a chatter-free milling process. Int. J. Mach. Tools Manuf., 72 (2013) 11-15.

6. X.M. Zhang, L. Zhu, D. Zhang, et al. Numerical robust optimization of spindle speed for milling process with uncertainties, Int. J. Mach. Tools Manuf., 61 (2012): 9-19. 
7. H. Xiao, D. Ren, S. Wang. EDM machining quality control and parameters optimization. Int. J. Adv. Manuf. Technol., 89 (2017) 1307-1315.

8. Y. Li, Q. Liu. Service-oriented research on multipass milling parameters optimization for green and high efficiency. J. Mech. Eng.,51 (2015) 89-98

9. H.R. Cao, X.F Chen. Z.J He. Modeling of spindleprocess interaction and cutting parameters optimization in high-speed milling. J. Mech. Eng., 49 (2013) 161-166.

10. D. Fratila, C. Caizar. Application of Taguchi method to selection of optimal lubrication and cutting conditions in face milling of AlMg3. J. Clean. Prod., 19 (2011) 640-645.

11. E. Budak, A. Tekeli, Maximizing chatter free material removal rate in milling through optimal selection of axial and radial depth of cut pairs, CIRP Annals-Manuf. Technol. 54 (2005) 353-356.

12. E. Graham, M. Mehrpouya, S.S. Park. Robust prediction of chatter stability in milling based on the analytical chatter stability. J. Manuf. Processes, 15 (2013) 508-517.

13. S.S. Park Y.M. Qin. Robust regenerative chatter stability in machine tools, Int. J. Adv. Manuf. Technol., 33 (2007) 389- 402.

14. P. Yi, K. Wei, X. Kong, et al. Cumulative PSOKriging model for slope reliability analysis, Probabilist Eng. Mech, 39 (2015) 39-45. 